PROCEEDINGS OF THE

AMERICAN MATHEMATICAL SOCIETY

Volume 135, Number 6, June 2007, Pages 1701-1708

S 0002-9939(06)08665-5

Article electronically published on November 13, 2006

\title{
WEYL TYPE THEOREMS AND HYPERCYCLIC OPERATORS II
}

\author{
XIAOHONG CAO
}

(Communicated by N. Tomczak-Jaegermann)

\begin{abstract}
In this note, the relation between hypercyclic operator matrices (or supercyclic operator matrices) and the operator matrices which satisfy Weyl type theorems is discussed. Also, using a variant of the essential approximate point spectrum, we give the necessary and sufficient conditions for $A$ for which a-Browder's theorem or a-Weyl's theorem holds.
\end{abstract}

Throughout this note, let $H$ and $K$ be complex, separable, infinite-dimensional Hilbert spaces, let $B(H, K)$ denote the set of bounded linear operators from $H$ to $K$, and abbreviate $B(H, H)$ to $B(H)$. We recall 1 the hypercyclic and supercyclic operators of Hilden and Wallen ([7]) and Kitai $([8]): T \in H C(H)$ is hypercyclic if it has a hypercyclic vector $x \in H$ for which $\left\{T^{n} x: n \in \mathbb{N}\right\}$ are dense in $H$, and supercyclic if the scalar multiples of $\left\{T^{n} x: n \in \mathbb{N}\right\}$ are dense in $H$. Weyl's theorem has been studied by many authors ([2], 3], etc.). In this note, we study the hypercyclicity (supercyclicity) for operator matrices and give the relations between hypercyclic (supercyclic) operator matrices and the operator matrices for which Weyl type theorems hold. We continue the notation and definitions of paper 11. Let

$$
\sigma_{2}(A)=\pi_{0}(A) \backslash a c c \sigma_{e a}(A) .
$$

Recalling Theorem 3.3 and Corollary 3.4 from [1, we get:

Theorem 1.1. Suppose $\sigma_{2}(A)=\emptyset$ and suppose $\sigma(A)=\sigma_{a}(A)$ is connected. If $f \in H(A)$ such that $\left|f\left(\lambda_{0}\right)\right|=1$ for some $\lambda_{0} \in \sigma(A)$, then $f(A) \in \overline{H C(H)}$.

Corollary 1.2. If Weyl's theorem holds for A (or a-Weyl's theorem holds for A), then

(1) $A \in \overline{H C(H)} \Longleftrightarrow \sigma(A)=\sigma_{a}(A), \sigma_{2}(A)=\emptyset$, and $\sigma(A) \cup \partial \mathrm{D}$ is connected;

(2) $A \in \overline{S C(H)} \Longleftrightarrow \sigma(A)=\sigma_{a}(A), \sigma_{2}(A)=\emptyset$ or $\sigma_{2}(A)=\{\lambda\}$, where $\lambda \neq 0$ and $A-\lambda I$ is Browder, and both $\sigma(A) \cup \partial\left(\mathrm{r}_{1} \mathrm{D}\right)$ and $\sigma_{w}(A) \cup \partial\left(\mathrm{r}_{2} \mathrm{D}\right)$ are connected for some $r_{1} \geq 0, r_{2} \geq 0$.

The present Theorem 1.1 is an extension to Theorem 3.3 of [1. In fact, suppose that the conditions in Theorem 3.3 in 1 hold; since $\sigma_{2}(A) \subseteq \sigma(A) \backslash \sigma_{1}(A)$ and $\sigma(A) \backslash \sigma_{a}(A) \subseteq \sigma(A) \backslash \sigma_{1}(A)$, where $\sigma_{1}(A)$ is defined in [1, it follows that $\sigma_{2}(A)=\emptyset$

Received by the editors October 24, 2005 and, in revised form, December 21, 2005 and January $24,2006$.

2000 Mathematics Subject Classification. Primary 47A15, 47A53, 47A55.

Key words and phrases. a-Browder's theorem, a-Weyl's theorem, Weyl's theorem, Browder's theorem, hypercyclic operator.

(C)2006 American Mathematical Society Reverts to public domain 28 years from publication 
and $\sigma(A)=\sigma_{a}(A)$ is connected. Also the present Corollary 1.2 is an extension to Corollary 3.4 of 1 .

Example. Let $H=\ell_{p}(p \geq 1) ; A \in B(H)$ is defined by

$$
A\left(x_{1}, x_{2}, x_{3}, \cdots\right)=\left(x_{2}, x_{3}, x_{4}, \cdots\right) .
$$

Then

(1) $\sigma(A)=\sigma_{w}(A)=\mathrm{D}$ and $\pi_{00}(A)=\emptyset$, which means that Weyl's theorem holds for $A$;

(2) $\sigma(A)=\sigma_{a}(A)=\mathrm{D}$ is connected and $\sigma_{2}(A)=\emptyset$. Hence

(a) $A \in \overline{H C(H)}$.

(b) For every $f \in H(A)$, there exists $c \in \mathbb{C}$ such that $c f(A) \in \overline{H C(H)}$. For instance, $\cos (A) \in \overline{H C(H)}$ and $\sin \left(\frac{\pi}{2} A\right) \in \overline{H C(H)}$.

(c) For every $f \in H(A), f(A) \in \overline{S C(H)}$.

Let $A^{*}$ denote the conjugate of $A$. In general, $A \in \overline{H C(H)}$ does not imply $A^{*} \in \overline{H C(H)}$. For example, let $A \in B\left(\ell_{2}\right)$, be defined by $A\left(x_{1}, x_{2}, x_{3}, \cdots\right)=$ $\left(x_{2}, x_{4}, x_{6}, \cdots\right)$; then $A \in \overline{H C\left(\ell_{2}\right)}$ while $A^{*} \notin \overline{H C\left(\ell_{2}\right)}$. But:

Corollary 1.3. If Weyl's theorem holds for A (or a-Weyl's theorem holds for A), then

(1) $A \in \overline{H C(H)}$ and $A^{*} \in \overline{H C(H)} \Longleftrightarrow \sigma(A)=\sigma_{a}(A)=\sigma_{S F_{-}}(A), \sigma_{2}(A)=\emptyset$, and $\sigma(A) \cup \partial \mathrm{D}$ is connected;

(2) $A \in \overline{S C(H)}$ and $A^{*} \in \overline{S C(H)} \Longleftrightarrow \sigma(A)=\sigma_{a}(A)=\sigma_{S F_{-}}(A)$, or $\sigma(A)=$ $\sigma_{a}(A)=\sigma_{S F_{-}}(A) \cup\{\lambda\}, \sigma_{2}(A)=\emptyset$ or $\sigma_{2}(A)=\{\lambda\}$, where $\lambda \neq 0$ and $A-\lambda I$ is Browder, and both $\sigma(A) \cup \partial\left(r_{1} \mathrm{D}\right)$ and $\sigma_{w}(A) \cup \partial\left(r_{2} \mathrm{D}\right)$ are connected for some $r_{1} \geq 0, r_{2} \geq 0$.

The following theorem give the necessary and sufficient conditions for $T$ for which a-Weyl's theorem holds.

Theorem 1.4. $a$-Weyl's theorem holds for $A \Longleftrightarrow \sigma_{2}(A) \subseteq \overline{P_{a b}(A)}$, where $P_{a b}(A)=$ $\sigma_{a}(A) \backslash \sigma_{a b}(A)$.

Proof. Suppose a-Weyl's theorem holds for $A$. Let $\lambda_{0} \in \sigma_{2}(A)$. Then $0<$ $\operatorname{dim} N\left(A-\lambda_{0} I\right)<\infty$, and there exists $\epsilon>0$ such that $A-\lambda I \in S F_{+}^{-}(H)$ if $0<\left|\lambda-\lambda_{0}\right|<\epsilon$. Since a-Weyl's theorem holds for $A$, it follows that $A-\lambda I$ has finite ascent. It induces that $\lambda \in P_{a b}(A) \cup \rho_{a}(A)$ if $0<\left|\lambda-\lambda_{0}\right|<\epsilon$. Then $\lambda_{0} \in \operatorname{acc}\left[P_{a b}(A)\right] \cup$ iso $\sigma_{a}(A)$. If $\lambda_{0} \in$ iso $\sigma_{a}(A)$, since $0<\operatorname{dim} N\left(A-\lambda_{0} I\right)<\infty$, $\lambda_{0} \in \pi_{00}^{a}(A)$. But since a-Weyl's theorem holds for $A, \lambda_{0} \in \sigma_{a}(A) \backslash \sigma_{e a}(A)$ and $A-\lambda_{0} I$ has finite ascent. Then $\lambda_{0} \in P_{a b}(A)$. From the preceding proof, we know that $\sigma_{2}(A) \subseteq a c c\left[P_{a b}(A)\right] \cup P_{a b}(A)=\overline{P_{a b}(A)}$.

For the converse, suppose that $\sigma_{2}(A) \subseteq \overline{P_{a b}(A)}$. Let $\lambda_{0} \in \sigma_{a}(A) \backslash \sigma_{e a}(A)$; then $\lambda_{0} \in \sigma_{2}(A) \subseteq \overline{P_{a b}(A)}$. We claim that $\lambda_{0} \notin$ acc[iso $\left.\sigma_{a}(A)\right]$. In fact, if $\lambda_{0} \in \operatorname{acc}\left[\right.$ iso $\left.\sigma_{a}(A)\right]$, since $A-\lambda_{0} I \in S F_{+}^{-}(H)$, using the punctured neighborhood theorem, there exists $\epsilon>0$ such that $A-\lambda I \in S F_{+}^{-}(H)$ and

$$
N(A-\lambda I) \subseteq \bigcap_{n=1}^{\infty} R\left[(A-\lambda I)^{n}\right]
$$

if $0<\left|\lambda-\lambda_{0}\right|<\epsilon$, and there exists $\lambda_{1} \in$ iso $\sigma_{a}(A)$ and $0<\left|\lambda_{1}-\lambda_{0}\right|<\epsilon$. Then $A$ has the single-valued extension property at $\lambda_{1}$. Theorem 11 in $[5]$ tells us that 
$A-\lambda_{1} I$ has finite ascent. Thus $N\left(A-\lambda_{1} I\right)=N\left(A-\lambda_{1} I\right) \cap \bigcap_{n=1}^{\infty} R\left[\left(A-\lambda_{1} I\right)^{n}\right]=$ $\{0\}$ (10, Lemma 3.4]), which means that $A-\lambda_{1} I$ is bounded from below. It is in contradiction to the fact that $\lambda_{1} \in$ iso $\sigma_{a}(A)$. Then $\lambda_{0} \in P_{a b}(A)$, hence $\lambda_{0} \in \pi_{00}^{a}(A)$. Conversely, let $\lambda_{0} \in \pi_{00}^{a}(A)$; then $\lambda_{0} \in \sigma_{2}(A) \subseteq \overline{P_{a b}(A)}$. Therefore $\lambda_{0} \in P_{a b}(A)$ because $\lambda_{0} \notin$ acc $\left[\right.$ iso $\left.\sigma_{a}(A)\right]$. Thus $\lambda_{0} \in \sigma_{a}(A) \backslash \sigma_{e a}(A)$. Now we have proved that $\sigma_{a}(A) \backslash \sigma_{e a}(A)=\pi_{00}^{a}(A)$, which means that a-Weyl's theorem holds for A.

Example. An operator $A \in B(X)$ is said to be p-hyponormal if $\left(A A^{*}\right)^{p} \leq\left(A^{*} A\right)^{p}$, and an operator $A \in B(X)$ is said to be M-hyponormal if there exists a positive real number $M$ such that

$$
M\|(A-\lambda I) x\| \geq\left\|(A-\lambda I)^{*} x\right\| \quad \text { for all } \lambda \in \mathbb{C} \text { for all } x \in H .
$$

If $p=1$, then $A$ is called simply hyponormal, and it is equivalent to the case where $M=1$.

First let us introduce some well-known facts of p-hyponormal and M-hyponormal operators:

(a) If $A$ is p-hyponormal or M-hyponormal, then so is $A-\lambda I$ for each $\lambda \in \mathbb{C}$.

(b) If $A$ is p-hyponormal or M-hyponormal and $E \subseteq X$ is invariant under $A$, then $\left.A\right|_{E}$ is p-hyponormal or M-hyponormal.

(c) If $A$ is p-hyponormal or M-hyponormal and quasinilpotent, then $A$ is nilpotent.

(d) If $A$ is p-hyponormal or M-hyponormal, then $A$ has finite ascent.

Suppose $A^{*} \in B(X)$ is a p-hyponormal or M-hyponormal operator; then the a-Weyl's theorem holds for $A$.

In fact, from Theorem 1.4, we need to prove that $\sigma_{2}(A) \subseteq \overline{P_{a b}(A)}$. Suppose $\lambda_{0} \in \sigma_{2}(A)$. Then $0<\operatorname{dim} N\left(A-\lambda_{0} I\right)<\infty$ and there exists $\epsilon>0$ such that $A-\lambda I \in S F_{+}^{-}(H)$ if $0<\left|\lambda-\lambda_{0}\right|<\epsilon$. Thus $A^{*}-\bar{\lambda} I$ is lower semi-Fredholm with $\operatorname{ind}\left(A^{*}-\bar{\lambda} I\right) \geq 0$. Since $A^{*}-\bar{\lambda} I$ has finite ascent, it follows that $\operatorname{ind}\left(A^{*}-\bar{\lambda} I\right) \leq 0$, which means that $A^{*}-\bar{\lambda} I$ is Weyl. Using the fact that $A^{*}-\bar{\lambda} I$ has finite ascent, we get that $A^{*}-\bar{\lambda} I$ is Browder, thus $A-\lambda I$ is Browder if $0<\left|\lambda-\lambda_{0}\right|<\epsilon$. Then $\lambda_{0} \in \operatorname{acc}\left[P_{a b}(A)\right] \cup i s o \sigma_{a}(A)$. If $\lambda_{0} \in i \operatorname{so\sigma }(A)$, we will prove that $\lambda_{0} \in P_{a b}(A)$. By $\overline{\lambda_{0}} \in$ iso $\sigma\left(A^{*}\right)$, using the spectral projection, we can represent $A^{*}$ as the direct sum

$$
A^{*}=A_{1}+A_{2}, \quad \text { where } \sigma\left(A_{1}\right)=\left\{\overline{\lambda_{0}}\right\} \text { and } \sigma\left(A_{2}\right)=\sigma\left(A^{*}\right) \backslash\left\{\overline{\lambda_{0}}\right\} .
$$

Since $A_{1}-\overline{\lambda_{0}} I$ is hyponormal and quasinilpotent, if follows that $A_{1}-\overline{\lambda_{0}} I$ is nilpotent. It induces that $A_{1}-\overline{\lambda_{0}} I$ has finite ascent and finite descent. But since $A_{2}-\overline{\lambda_{0}} I$ is invertible, we know that $A^{*}-\overline{\lambda_{0}} I$ has finite ascent and finite descent. Then $A-\lambda_{0} I$ has finite ascent and finite descent. Using the fact that $0<\operatorname{dim} N\left(A-\lambda_{0} I\right)<\infty$, we can induce that $A-\lambda_{0} I$ is Browder. Then $\lambda_{0} \in P_{a b}(A)$. Thus $\lambda_{0} \in \overline{P_{a b}(A)}$. Now we get $\sigma_{2}(A) \subseteq \overline{P_{a b}(A)}$, which means that the a-Weyl's theorem holds for $A$.

If $A^{*}$ is a p-hyponormal or M-hyponormal operator, then $\sigma(A)=\sigma_{a}(A)$. Suppose $\sigma(A)$ is connected; we claim that $\sigma_{2}(A)=\emptyset$. If not, let $\lambda_{0} \in \sigma_{2}(A)$. Then $A-\lambda I \in$ $S F_{+}^{-}(H)$ if $0<\left|\lambda-\lambda_{0}\right|$ is sufficiently small. Thus $A-\lambda I$ is Weyl. Since a-Weyl's theorem implies Weyl's theorem, it follows that $A-\lambda I$ is Browder. Using the fact that $\sigma(A)$ is connected, we know $i \operatorname{so} \sigma(A)=\emptyset$. Then $\lambda_{0} \in \pi_{00}^{a}(A)$. But since a-Weyl's theorem holds for $A, A-\lambda_{0} I \in S F_{+}^{-}(H)$ and $A-\lambda_{0} I$ has finite ascent. 
Then $A-\lambda_{0} I$ is Browder. It is in contradiction to the fact that $i \operatorname{so} \sigma(A)=\emptyset$. Thus $\sigma_{2}(A)=\emptyset$.

From Corollary 1.2 and Theorem 1.1, if $A^{*}$ is a p-hyponormal or M-hyponormal operator we have:

(1) $A \in \overline{H C(H)} \Longleftrightarrow \sigma_{2}(A)=\emptyset$ and $\sigma(A) \cup \partial \mathrm{D}$ is connected.

(2) If $\sigma(A)$ is connected, then for any $f \in H(A)$, there exists $c \neq 0$ such that $c f(A) \in \overline{H C(H)}$.

The study of upper triangular operator matrices arises naturally from the following fact: if $T$ is a Hilbert space operator and $M$ is an invariant subspace for $T$, then $T$ has the following $2 \times 2$ upper triangular operator matrix representation:

$$
T=\left(\begin{array}{ll}
* & * \\
0 & *
\end{array}\right): \quad M \oplus M^{\perp} \longrightarrow M \oplus M^{\perp},
$$

and one way to study operators is to see them as entries of simpler operators. When $A \in B(H)$ and $B \in B(K)$ are given, we denote by $M_{C}$ an operator acting on $H \oplus K$ of the form

$$
M_{C}=\left(\begin{array}{cc}
A & C \\
0 & B
\end{array}\right),
$$

where $C \in B(K, H)$. If $C=0$, let $M_{0}=\left(\begin{array}{cc}A & 0 \\ 0 & B\end{array}\right)$. In the following, using the spectrum set $\sigma_{2}(\cdot)$, we show the relation between hypercyclic operator matrices and the operator matrices which satisfy Weyl type theorems.

Theorem 1.5. Suppose $\sigma(A)=\sigma_{a}(A)$ and $\sigma_{2}(A)=\emptyset \quad\left(\right.$ or $\sigma_{2}(A)=\{\lambda\}$, where $T-\lambda I$ is Browder). Then for any $B \in B(K)$ and $C \in B(K, H)$,

(1) $\left(\begin{array}{cc}A & 0 \\ 0 & B\end{array}\right) \in \overline{H C(H \oplus K)} \Longrightarrow\left(\begin{array}{cc}A & C \\ 0 & B\end{array}\right) \in \overline{H C(H \oplus K)}$;

(2) $\quad\left(\begin{array}{cc}A & 0 \\ 0 & B\end{array}\right) \in \overline{S C(H \oplus K)} \Longrightarrow\left(\begin{array}{cc}A & C \\ 0 & B\end{array}\right) \in \overline{S C(H \oplus K)}$.

Proof. Suppose $M_{0} \in \overline{H C(H \oplus K)}$.

(1) $\sigma_{w}\left(M_{C}\right)=\sigma_{w}\left(M_{0}\right)$ for every $C \in B(K, H)$. Hence $\sigma_{w}\left(M_{C}\right) \cup \partial \mathrm{D}=$ $\sigma_{w}\left(M_{0}\right) \cup \partial \mathrm{D}$ is connected.

$\sigma_{w}\left(M_{C}\right) \subseteq \sigma_{w}\left(M_{0}\right)$ is clear. For the converse inclusion, let $M_{C}-\lambda_{0} I$ be Weyl. Then $A-\lambda_{0} I$ is upper semi-Fredholm, $B-\lambda_{0} I$ is lower semi-Fredholm and $A-\lambda_{0} I$ is Fredholm if and only if $B-\lambda_{0} I$ is Fredholm. We claim that $A-\lambda_{0} I$ is Fredholm. In fact, if $N\left(A-\lambda_{0} I\right)=\{0\}, A-\lambda_{0} I$ is bounded from below and hence $A-\lambda_{0} I$ is invertible because $\sigma(A)=\sigma_{a}(A)$. If $N\left(A-\lambda_{0} I\right) \neq\{0\}$, since $\sigma_{2}(A)=\emptyset$, it follows that the $\operatorname{dim}\left(A^{*}-\lambda_{0} I\right)<\infty$, which means that $A-\lambda_{0} I$ is Fredholm. Then $B-\lambda_{0} I$ is Fredholm. But since $M_{C}-\lambda_{0} I=\left(\begin{array}{cc}I & 0 \\ 0 & B-\lambda_{0} I\end{array}\right)\left(\begin{array}{cc}I & C \\ 0 & I\end{array}\right)\left(\begin{array}{cc}A-\lambda_{0} I & 0 \\ 0 & I\end{array}\right)$, we know that $M_{0}-\lambda_{0} I$ is Weyl. Hence $\sigma_{w}\left(M_{C}\right)=\sigma_{w}\left(M_{0}\right)$ for any $C \in B(K, H)$. Then $\sigma_{w}\left(M_{C}\right) \cup \partial \mathrm{D}=\sigma_{w}\left(M_{0}\right) \cup \partial \mathrm{D}$ is connected.

(2) $\sigma_{b}\left(M_{C}\right)=\sigma\left(M_{C}\right)$ for any $C \in B(K, H)$.

Let $M_{C}-\lambda_{0} I$ be Browder. From the proof (1) in this theorem, we know that $M_{0}-\lambda_{0} I$ is Weyl, $A-\lambda_{0} I$ has finite ascent and $B-\lambda_{0} I$ has finite descent. Since $\sigma_{2}(A)=\emptyset$ and $A-\lambda_{0} I$ has finite ascent, it induces that $A-\lambda_{0} I$ is Browder. Then $B-\lambda_{0}$ is Weyl with finite descent. Hence $B-\lambda_{0} I$ is Browder, too. Thus $M_{0}-\lambda_{0} I$ is Browder. But the fact that $M_{0} \in \overline{H C(H \oplus K)}$ tells us that $\sigma\left(M_{0}\right)=\sigma_{b}\left(M_{0}\right)$. Then $M_{0}-\lambda_{0} I$ is invertible. Therefore $M_{C}-\lambda_{0} I$ is invertible. 
(3) For each $C \in B(K, H)$, ind $\left(M_{C}-\lambda I\right) \geq 0$ for any $\lambda \in \rho_{S F}\left(M_{C}\right)$.

If not, let $\lambda \in \rho_{S F}\left(M_{C}\right)$ with $\operatorname{ind}\left(M_{C}-\lambda I\right)<0$. Then $A-\lambda I$ is upper semiFredholm. From the proof (1) in this theorem, we know that $A-\lambda I$ is Fredholm. Theorem 2.1 in 4 implies that $B-\lambda I$ is upper semi-Fredholm. Then $M_{0}-\lambda I$ is upper semi-Fredholm with $\operatorname{ind}\left(M_{0}-\lambda I\right)=\operatorname{ind}\left(M_{C}-\lambda I\right)<0$. This is in contradiction to the fact that $M_{0} \in \overline{H C(H \oplus K)}$ [6. Lemma 2.1]. Using ([6, Theorem 2.1 and Theorem 3.3]), we know that $M_{C} \in \overline{H C(H \oplus K)}$ for any $C \in B(K, H)$.

If $M_{0} \in \overline{S C(H \oplus K)}$, similar to the case of $M_{0} \in \overline{H C(H \oplus K)}$, we can prove the result.

We also can prove: If $\sigma(A)=\sigma_{a}(A)$ and $\sigma_{2}(A)=\emptyset$ (or $\sigma_{2}(A)=\{\lambda\}$, where $T-\lambda I$ is Browder), then for any $B \in B(K)$ and $C \in B(K, H)$,

(a) Browder's theorem holds for $\left(\begin{array}{cc}A & 0 \\ 0 & B\end{array}\right) \Longrightarrow$ Browder's theorem holds for $\left(\begin{array}{cc}A & C \\ 0 & B\end{array}\right)$

(b) a-Browder's theorem holds for $\left(\begin{array}{cc}A & 0 \\ 0 & B\end{array}\right) \Longrightarrow$ a-Browder's theorem holds for $\left(\begin{array}{cc}A & C \\ 0 & B\end{array}\right)$.

Suppose $\sigma(A)=\sigma_{a}(A), \sigma_{2}(A)=\emptyset$, and $\sigma(B)=\sigma_{a}(B), \sigma_{2}(B)=\emptyset$. Then $\sigma_{a}\left(M_{0}\right)=\sigma_{w}\left(M_{0}\right)=\sigma_{b}\left(M_{0}\right)=\sigma\left(M_{0}\right)$ and $\pi_{00}\left(M_{0}\right)=\emptyset$. This means Weyl's theorem holds for $M_{0}$. Corollary 1.2 tells us that $M_{0} \in \overline{H C(H \oplus K)} \Longleftrightarrow \sigma\left(M_{0}\right) \cup$ $\partial \mathrm{D}$ is connected and $M_{0} \in \overline{S C(H \oplus K)} \Longleftrightarrow \sigma\left(M_{0}\right) \cup \partial(\mathrm{rD})$ is connected for some $r \geq 0$. Using Theorem 1.5, we know that:

Corollary 1.6. Suppose $\sigma(A)=\sigma_{a}(A), \sigma_{2}(A)=\emptyset$, and $\sigma(B)=\sigma_{a}(B), \sigma_{2}(B)=\emptyset$, then:

(1) $M_{C} \in \overline{H C(H \oplus K)}$ for any $C \in B(K, H) \Longleftrightarrow \sigma\left(M_{0}\right) \cup \partial \mathrm{D}$ is connected;

(2) $M_{C} \in \overline{S C(H \oplus K)}$ for any $C \in B(K, H) \Longleftrightarrow \sigma\left(M_{0}\right) \cup \partial(r \mathrm{D})$ is connected for some $r \geq 0$.

In this case, a-Browder's theorem holds for $M_{C}$ for any $C \in B(K, H)$.

In Corollary 1.6, if $\sigma(A)=\sigma_{a}(A)=\sigma_{S F_{-}}(A), \sigma_{2}(A)=\emptyset$, and $\sigma(B)=\sigma_{a}(B)=$ $\sigma_{S F_{-}}(B), \sigma_{2}(B)=\emptyset$, then

(1) $M_{C} \in \overline{H C(H \oplus K)}$ and $M_{C}^{*} \in \overline{H C(H \oplus K)}$ for any $C \in B(K, H) \Longleftrightarrow$ $\sigma\left(M_{0}\right) \cup \partial \mathrm{D}$ is connected;

(2) $\quad M_{C} \in \overline{S C(H \oplus K)}$ and $M_{C}^{*} \in \overline{S C(H \oplus K)}$ for any $C \in B(K, H) \Longleftrightarrow$ $\sigma\left(M_{0}\right) \cup \partial(r \mathrm{D})$ is connected for some $r \geq 0$.

We call $A$ isoloid if every isolated point of $\sigma(A)$ is an eigenvalue of $A$. If $A \in$ $\overline{H C(H)}$ or $A \in \overline{S C(H)}$ and if $A$ is isoloid and Weyl's theorem holds for $A$, then $\sigma(A)=\sigma_{a}(A)$ and $\sigma_{2}(A)=\emptyset$ (or $\sigma_{2}(A)=\{\lambda\}$, where $A-\lambda I$ is Browder) (Corollary 1.2). Then the result in Theorem 1.5 holds. In additional, if $\sigma_{2}(B)=\emptyset$, we have:

Corollary 1.7. Suppose $A \in \overline{H C(H)}$ and $\sigma_{2}(B)=\emptyset$. If $A$ is isoloid and Weyl's theorem holds for $A$, then the following statements are equivalent:

(1) $\sigma_{2}\left(M_{0}\right)=\emptyset$;

(2) $a$-Weyl's theorem holds for $M_{0}$;

(3) $a$-Weyl's theorem holds for $M_{C}$ for any $C \in B(K, H)$;

(4) a-Browder's theorem holds for $M_{0}$;

(5) a-Browder's theorem holds for $M_{C}$ for any $C \in B(K, H)$;

(6) $\sigma_{2}\left(M_{C}\right)=\emptyset$ for any $C \in B(K, H)$. 
In this case, we have that:

(a) If $M_{0} \in \overline{H C(H \oplus K)}$, then $M_{C} \in \overline{H C(H \oplus K)}$ for any $C \in B(K, H)$.

(b) If $M_{0} \in \overline{S C(H \oplus K)}$, then $M_{C} \in \overline{S C(H \oplus K)}$ for any $C \in B(K, H)$.

Proof. $(1) \Longrightarrow(2)$. Since $\sigma_{2}\left(M_{0}\right) \subseteq \overline{P_{a b}\left(M_{0}\right)}$, from Theorem 1.4, we know that aWeyl's theorem holds for $M_{0}$.

$(2) \Longrightarrow(1)$. If there exists $\lambda_{0} \in \sigma_{2}\left(M_{0}\right)$, then $0<\operatorname{dim} N\left(M_{0}-\lambda_{0} I\right)<\infty$ and there exists $\epsilon>0$ such that $M_{0}-\lambda I \in S F_{+}^{-}(H \oplus K)$ if $0<\left|\lambda-\lambda_{0}\right|<\epsilon$. So $\operatorname{dim} N\left(A-\lambda_{0} I\right)<\infty$ and $\operatorname{dim} N\left(B-\lambda_{0} I\right)<\infty$. Since a-Weyl's theorem holds for $M_{0}$, it implies that $M_{0}-\lambda I$ has finite ascent. Then $A-\lambda I \in S F_{+}^{-}(H)$ and $B-\lambda I \in S F_{+}^{-}(K)$ and both $A-\lambda I$ and $B-\lambda I$ have finite ascent. Using the condition $A \in \overline{H C(H)}$ and $\sigma_{2}(B)=\emptyset$, we know that $A-\lambda_{0} I$ is invertible and $B-\lambda_{0} I$ is bounded from below. Then $N\left(M_{0}-\lambda_{0} I\right)=N\left(A-\lambda_{0} I\right) \oplus N\left(B-\lambda_{0} I\right)=$ $\{0\}$. This is a contradiction. Then $\sigma_{2}\left(M_{0}\right)=\emptyset$.

$(2) \Longrightarrow(3)$. We need to show that $\sigma_{a}\left(M_{C}\right)=\sigma_{e a}\left(M_{C}\right)$ and $\pi_{00}^{a}\left(M_{C}\right)=\emptyset$. Then $\sigma_{a}\left(M_{C}\right) \backslash \sigma_{e a}\left(M_{C}\right)=\pi_{00}^{a}\left(M_{C}\right)$, which means that a-Weyl's theorem holds for $M_{C}$.

Let $M_{C}-\lambda_{0} I \in S F_{+}^{-}(H \oplus K)$; from the proof in Theorem 1.5, we know that $M_{0}-\lambda_{0} I \in S F_{+}^{-}(H \oplus K)$. Since a-Weyl's theorem holds for $M_{0}$, it induces that $A-\lambda_{0} I$ and $B-\lambda_{0} I$ have finite ascent. Then $\operatorname{ind}\left(A-\lambda_{0} I\right) \leq 0$. But since $A \in \overline{H C(H)}, \operatorname{ind}\left(A-\lambda_{0} I\right) \geq 0$. Then $A-\lambda_{0} I$ is Weyl with finite ascent. Thus $A-\lambda_{0} I$ is Browder, and therefore $A-\lambda_{0} I$ is invertible and $\operatorname{ind}\left(B-\lambda_{0} I\right) \leq 0$. The condition $\sigma_{2}(B)=\emptyset$ implies that $B-\lambda_{0} I$ is bounded from below. Since $N\left(M-\lambda_{0} I\right) \subseteq\left(A-\lambda_{0} I\right)^{-1}\left[C N\left(B-\lambda_{0} I\right)\right] \oplus N\left(B-\lambda_{0} I\right)$, it induce that $M_{C}-\lambda_{0} I$ is bounded from below. Then $\sigma_{a}\left(M_{C}\right)=\sigma_{e a}\left(M_{C}\right)$ for any $C \in B(K, H)$. If there exists $\lambda_{0} \in \pi_{00}^{a}\left(M_{C}\right)$, then $0<\operatorname{dim} N\left(M_{C}-\lambda_{0} I\right)<\infty$ and there exists $\epsilon>0$ such that $M_{C}-\lambda I$ is bounded from below if $0<\left|\lambda-\lambda_{0}\right|<\epsilon$. Then $A-\lambda I$ is bounded from below. Since $\sigma(A)=\sigma_{a}(A)$, it follows that $A-\lambda I$ is invertible, hence $B-\lambda I$ is bounded from below, which means that $\lambda_{0} \in i \operatorname{so\sigma } \sigma_{a}\left(M_{0}\right)$ and $\lambda_{0} \in i \operatorname{so\sigma }(A) \cup \rho(A)$. Since $\operatorname{dim} N\left(A-\lambda_{0} I\right) \leq \operatorname{dim} N\left(M_{C}-\lambda_{0} I\right)<\infty$ and $A$ is isoloid, it implies that $\lambda_{0} \in \pi_{00}(A) \cup \rho(A)$. The fact that Weyl's theorem holds for $A$ and $\sigma(A)=\sigma_{b}(A)$ induces the fact that $A-\lambda_{0} I$ is invertible. From the proof of Theorem 2.4 in 9, we know that $\operatorname{dim} N\left(B-\lambda_{0} I\right)<\infty$. But since $\sigma_{2}(B)=\emptyset$, there must have $\operatorname{dim} N\left(B-\lambda_{0} I\right)=\{0\}$. Then $N\left(M_{C}-\lambda_{0} I\right)=\{0\}$, which is in contradiction to the fact that $0<\operatorname{dim} N\left(M_{C}-\lambda_{0} I\right)<\infty$. Then $\sigma_{a}\left(M_{C}\right) \backslash \sigma_{e a}\left(M_{C}\right)=\pi_{00}^{a}\left(M_{C}\right)=\emptyset$, which means that a-Weyl's theorem holds for $M_{C}$ for any $C \in B(K, H)$.

$(3) \Longrightarrow(2)$. This is clear.

Since a-Weyl's theorem implies a-Browder's theorem, then $(2) \Longrightarrow(4)$ and $(3) \Longrightarrow(5)$ is clear. $(5) \Longrightarrow(4)$ and $(6) \Longrightarrow(1)$ are also clear.

$(5) \Longrightarrow(3)$. From the proof in $(2) \Longrightarrow(3)$, we know that $\pi_{00}^{a}\left(M_{C}\right)=\emptyset$. But since aBrowder's theorem holds for $M_{C}$, it follows that $\sigma_{a}\left(M_{C}\right) \backslash \sigma_{e a}\left(M_{C}\right) \subseteq \pi_{00}^{a}\left(M_{C}\right)=\emptyset$. Then $\sigma_{a}\left(M_{C}\right) \backslash \sigma_{e a}\left(M_{C}\right)=\pi_{00}^{a}\left(M_{C}\right)=\emptyset$, which means that a-Weyl's theorem holds for $M_{C}$ for every $C \in B(K, H)$.

$(1) \Longrightarrow(6)$. If there exists $\lambda_{0} \in \sigma_{2}\left(M_{C}\right)$, then $0<\operatorname{dim} N\left(M_{C}-\lambda_{0} I\right)<\infty$ and there exists $\epsilon>0$ such that $M_{C}-\lambda I \in S F_{+}^{-}(H \oplus K)$. From the preceding proof, $A-\lambda I$ is Fredholm and $B-\lambda I$ is upper semi-Fredholm. Then $M_{0}-\lambda I \in$ $S F_{+}^{-}(H \oplus K)$. Since $\sigma_{2}\left(M_{0}\right)=\emptyset$, it follows that $M_{0}-\lambda I$ is bounded from below, which implies that $M_{C}-\lambda I$ is bounded from below. Then $\lambda_{0} \in \pi_{00}^{a}\left(M_{C}\right)$. But from the preceding proof, we know that $\pi_{00}^{a}\left(M_{C}\right)=\emptyset$, a contradiction. 
Using Theorem 1.5, similar to the proof of Corollary 1.7, we can get that

Corollary 1.8. Suppose $\sigma(A)=\sigma_{a}(A)$ and $\sigma_{2}(A)=\emptyset$. If $A$ is isoloid and $M_{0} \in$ $\overline{H C(H \oplus K)}$, then the following statements are equivalent:

(1) $\sigma_{2}\left(M_{0}\right)=\emptyset$;

(2) $a$-Weyl's theorem holds for $M_{0}$;

(3) $a$-Weyl's theorem holds for $f\left(M_{0}\right)$ for any $f \in H\left(M_{0}\right)$;

(4) $a$-Weyl's theorem holds for $M_{C}$ for any $C \in B(K, H)$;

(5) for any $C \in B(K, H)$ and for every $f \in H\left(M_{C}\right)$, a-Weyl's theorem holds for $f\left(M_{C}\right)$;

(6) Weyl's theorem holds for $M_{0}$;

(7) Weyl's theorem holds for $M_{C}$ for any $C \in B(K, H)$;

(8) $\sigma_{2}\left(M_{0}\right)=\emptyset$ for any $C \in B(K, H)$.

For a-Browder's theorem, we have:

Theorem 1.9. a-Browder's theorem holds for $T \Longleftrightarrow \sigma_{2}(T) \subseteq \overline{i s o \sigma(T)}$.

Corollary 1.10. Suppose $\sigma(A)=\sigma_{a}(A)$ and $\sigma_{2}(A)=\emptyset$ (or $\sigma_{2}(A)=\{\lambda\}$, where $A-\lambda I$ is Browder $)$. If $M_{0} \in \overline{H C(H \oplus K)}$ or $M_{0} \in \overline{S C(H \oplus K)}$, then the following statements are equivalent:

(1) $\sigma_{2}\left(M_{0}\right) \subseteq i \operatorname{so\sigma }\left(M_{0}\right)$

(2) a-Browder's theorem holds for $M_{0}$;

(3) $a$-Browder's theorem holds for $M_{C}$ for any $C \in B(K, H)$;

(4) Browder's theorem holds for $M_{0}$;

(5) Browder's theorem holds for $M_{C}$ for any $C \in B(K, H)$;

(6) $\sigma_{2}\left(M_{C}\right) \subseteq i \operatorname{so\sigma }\left(M_{0}\right)$ for each $C \in B(K, H)$.

Example. Let $A, B \in B\left(\ell_{2}\right)$ be defined by

$$
\begin{gathered}
A\left(x_{1}, x_{2}, x_{3}, \cdots\right)=\left(x_{2}, x_{4}, x_{6}, \cdots\right), \\
B\left(x_{1}, x_{2}, x_{3}, \cdots\right)=\left(0, x_{1}, 0, x_{2}, 0, x_{3}, 0, \cdots\right) .
\end{gathered}
$$

Then $A$ is isoloid and a-Weyl's theorem holds for $A$, and $A \in \overline{H C(H)} ; \sigma_{2}(B)=\emptyset$.

Also, a straightforward calculation shows that $\sigma_{a}\left(M_{0}\right)=\sigma_{e a}\left(M_{0}\right)=\sigma_{a b}\left(M_{0}\right)=$ D. Then a-Browder's theorem holds for $M_{0}$. From Corollary 1.7, we know that for each $C \in B\left(\ell_{2}, \ell_{2}\right)$, a-Weyl's theorem holds for $M_{C}$.

Since $\sigma\left(M_{0}\right)=\sigma_{w}\left(M_{0}\right)=\sigma_{b}\left(M_{0}\right)=\mathrm{D}$ and $\rho_{S F}\left(M_{0}\right)=\mathbb{C} \backslash \sigma\left(M_{0}\right)=\rho\left(M_{0}\right)$, it follows that $M_{0} \in \overline{H C(H \oplus K)}$. Using Corollary 1.7, we know that $M_{C} \in$ $\overline{H C(H \oplus K)}$ for any $C \in B\left(\ell_{2}, \ell_{2}\right)$.

\section{ACKNOWLEDGEMENT}

We are grateful to the referees for helpful comments concerning this paper.

\section{REFERENCES}

[1] X.H.Cao, Weyl type theorem and hypercyclic operators, J. Math. Anal. Appl., 323(2006), 267-274.

[2] X.H.Cao, M.Z.Guo and B.Meng, Weyl spectra and Weyl's theorem, J. Math. Anal. Appl., 288(20) (2003), 758-767. MR2020195 (2004k:47002)

[3] X.H.Cao, Weyl's theorem for analytically hyponormal operators, L. Algeb. Appl., 405 (2005) 229-238. MR2148172 (2006d:47039) 
[4] X.H.Cao and B.Meng, Essential approximate point spectrum and Weyl's theorem for operator matrices, J. Math. Anal. Appl. 304 (2005), 759-771. MR2127605 (2005m:47005)

[5] J.K.Finch, The single valued extension property on a Banach space, Pacific. J. Math. 58 (1975), 61-69. MR0374985(51:11181)

[6] D.A.Herrero, Limits of hypercyclic and supercyclic operators, J. Functional Analysis 99 (1991), 179-190. MR1120920 (92g:47026)

[7] H.M.Hilden and L.J.Wallen, Some cyclic and non-cyclic vectors for certain operators, Indiana Univ. Math. J. 23 (1974), 557-565. MR0326452(48:4796)

[8] C.Kitai, Invariant closed sets for linear operators, Dissertation, Univ. of Toronto, 1982.

[9] W.Y.Lee, Weyl's theorem for operator matrices, Integr. Equ. Oper. Theory 32 (1998), 319331. MR 1652693 (99g:47023)

[10] A.E.Taylor, Theorems on ascent, descent, nullity and defect of linear operators, Math. Ann. 163 (1966) 18-49. MR0190759(32:8169)

College of Mathematics and Information Science, Shaanxi Normal University, Xi'an, 710062, People's Republic of China

E-mail address: xiaohongcao@snnu.edu.cn 